\title{
Taming Hamiltonian systems with balanced loss and gain via Lorentz interaction : General results and a case study with Landau Hamiltonian
}

\author{
Pijush K. Ghosh* \\ Department of Physics, Siksha-Bhavana, \\ Visva-Bharati University, \\ Santiniketan, PIN 731 235, India.
}

\begin{abstract}
The kinetic energy term of Hamiltonian systems with balanced loss and gain is not semipositive-definite, leading to instabilities at the classical as well quantum level. It is shown that an additional Lorentz interaction in the Hamiltonian allows the kinetic energy term to be semi-positive-definite and thereby, improving the stability properties of the system. Further, a consistent quantum theory admitting bound states may be obtained on the real line instead of Stoke wedges on the complex plane. The Landau Hamiltonian in presence of balanced loss and gain is considered for elucidating the general result. The kinetic energy term is semi-positive-definite provided the magnitude of the applied external magnetic field is greater than the magnitude of the 'analogous magnetic field' due to the loss-gain terms. It is shown that the classical particle moves on an elliptical orbit with a cyclotron frequency that is less than its value in absence of the loss-gain terms. The quantum system share the properties of the standard Landau Hamiltonian, but, with the modified cyclotron frequency. It is shown that the Hall current has non-vanishing components along the direction of the external uniform electric field and to its transverse direction. The Pauli equation in presence of balanced loss and gain is shown to be supersymmetric.
\end{abstract}

Keywords: Dissipative system, Hamiltonian formulation, Lorentz interaction, Landau Hamiltonian, Supersymmetry

PACS: 03.65.-w, 45.20.Jj, 11.30.Pb

\section{Contents}

1 Introduction

2 Formalism \& General result

2.1 Lorentz interaction \& a semi-positive definite $\mathcal{M} \ldots \ldots \ldots \ldots$

2.2 Representation of matrices . . . . . . . . . . . . . . . 8

2.3 Hiding the loss-gain terms . . . . . . . . . . . . . . . . . . 10

*email: pijushkanti.ghosh@visva-bharati.ac.in 
3 Landau Hamiltonian with balanced loss and gain 11

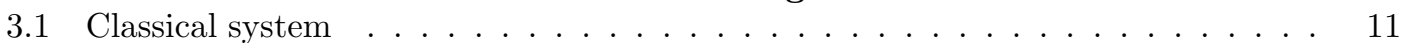

3.1 .1 Region-I . . . . . . . . . . . . . . . . . . 13

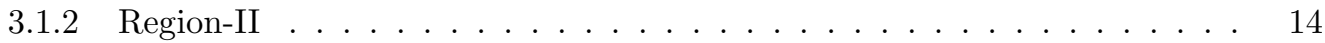

3.1.3 Region-III . . . . . . . . . . . . . . . . . . . . . 14

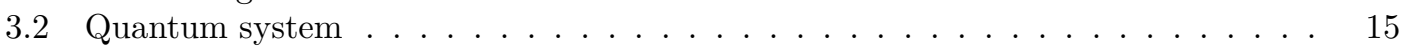

3.2 .1 Region-I . . . . . . . . . . . . . . . . . . . . . . . . . . . . . . . . .

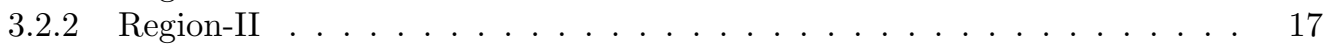

3.2 .3 Region-III . . . . . . . . . . . . . . . . . . 18

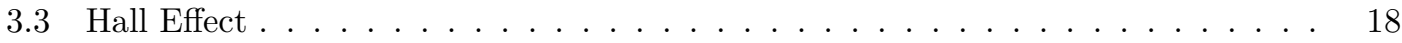

3.4 Spin, Pauli equation \& Supersymmetry . . . . . . . . . . . . . 19

4 Summary \& Discussions 20

5 Acknowledgments $\quad 22$

6 Appendix-A: Representation of $\mathcal{M}, R$ and $\mathcal{D} \quad 22$

6.1 Representation-I . . . . . . . . . . . . . . . . . . . . . . . . . . . . . . . . . .

6.2 Representations-II . . . . . . . . . . . . . . . . . . 22

\section{Introduction}

Hamiltonian systems with balanced loss and gain have received considerable interest 1, 2, 23, 4, 5, 6, 7, 8, 9, 10, in the literature due to its potential applications in various branches of physics. One of the major drawbacks of such Hamiltonian systems is that the kinetic energy term is not positive-definite. Consequently, stable classical solutions are obtained for specific choices of the potentials and that too, within restricted ranges of parameters. Further, a consistent quantum theory for such systems admitting bound states requires extensions of the eigen-value problem to the complex domain. The normalizability of eigenfunctions is obtained in specific Stoke wedges. Although such an extension of quantum mechanics is consistent from the viewpoint of axiomatic foundation of the subject 11, 12, no experimental support for such theories has been observed so far. A natural question one would like to pose at this juncture is whether or not a modification of Hamiltonian systems with balanced loss and gain is possible so that the kinetic energy term can be made semi-positive definite.

The purpose of this article is to show that the answer to the question posed above is in the affirmative and can be achieved by the inclusion of Lorentz interaction in the system. It may be noted in this regard that a vanishing trace of the matrix appearing in the quadratic form of momenta defining the kinetic energy term necessarily makes it non-positive-definite. All previous investigations on Hamiltonian systems with balanced loss and gain belong to this class [8, 9, 10, It is shown that a non-vanishing trace of this matrix requires the inclusion of non-dissipative velocity-dependent forces in the system with asymmetric coupling between the gain and loss degrees of freedom. The Lorentz force belongs to this class and its inclusion in the system raises the possibility of making the kinetic energy term positive-definite for some ranges in the parameter space. It is worth emphasizing here that the Lorentz force appears in diverse areas of science and the working principle of many devices are based on it. Thus, inclusion of Lorentz force in the study of Hamiltonian system with balanced loss and gain is only natural and may 
open up new avenues for theoretical understanding of these systems with possible technological applications.

The generic formulation of Hamiltonian system with space-dependent balanced loss and gain is modified appropriately to include Lorentz force in the system. This has been done for arbitrary number of particles and also for generic space-dependence of the gain-loss co-efficients. It has been shown that the inclusion of Lorentz force implies that the balancing of loss and gain is not necessarily in a pair-wise fashion and it may be achieved in several ways. This should be contrasted with all previous investigations on Hamiltonian systems with balanced loss and gain, where balancing is necessarily achieved in a pair-wise fashion. This allows more flexibility in constructing and controlling Hamiltonian systems with balanced loss and gain.

It is shown on general ground that Hamiltonian systems with balanced loss-gain and in presence of Lorentz force may be interpreted as defined in the background of a metric subjected to an effective external magnetic field, whose magnitude depends on the applied magnetic field related to the Lorentz force and an analogous magnetic field due to the loss-gain terms. The background metric is either Euclidean or pseudo-Euclidean, depending on the region in the parameter space in which it is defined. The metric is Euclidean, if the magnitude of the applied magnetic field is greater than the magnitude of the analogous magnetic field and pseudo-euclidean, otherwise. The specific signature of the pseudo-euclidean metric is model dependent. For the case of zero applied magnetic field, the known result that the background metric is only pseudo-Euclidean is recovered.

The Landau Hamiltonian [13] appears in the description of various physical phenomena, including quantum Hall effect and spintronics based devices. The Landau Hamiltonian with balanced loss and gain is considered as an example to elucidate the general result. There are three regions in the parameter-space corresponding to an Euclidean, a pseudo-Euclidean and a negative-definite metric. It is shown that the system for the case of Euclidean metric admits periodic solution with the cyclotron frequency determined in terms of the effective magnetic-field, thereby, with a reduced value compared to the case of Landau Hamiltonian without the loss-gain terms. Further, the circular orbit of the particle is deformed to an ellipse due to the presence of the loss-gain terms. The Hamiltonian is bounded from below. There are no stable solutions for the case of pseudo-Euclidean metric. The solutions for the case of a negative-definite metric share the same feature as in the case of Euclidean metric. However, the Hamiltonian is not bounded from below for this case.

The canonical quantization scheme is followed to obtain quantum Landau Hamiltonian with balanced loss and gain terms. The parameter space is again divided into three regions as in the case of corresponding classical system. For the case of Euclidean metric, all known results of the standard Landau Hamiltonian are reproduced with a modified expression for the cyclotron frequency in terms of the effective magnetic field. For the case of pseudo-Euclidean metric, no bound states are possible either on the real lines or by extending the problem to complex domain. For the case of negative definite metric, the Hamiltonian is bounded from above. The eigenfunctions of the Hamiltonian in this region are also eigenfunctions of the Hamiltonian for the case of Euclidean metric. However, the energy eigenvalues of the Hamiltonian in these two regions differ by an overall multiplication factor of -1 .

The Hall effect with balanced loss and gain is studied by including an external uniform electric field to the Landau Hamiltonian. It is shown that the Hall current has non-vanishing components along the direction of the applied electric field as well as to its transverse direction. The angle between the direction of the Hall current and that of the applied electric field depends on the gain-loss parameter. The result is valid at the classical as well as quantum level.

It is known that the Pauli equation has an underlying supersymmetry[14]. A similar investigation is carried out for the Landau Hamiltonian with balanced loss and gain by including an 
additional Zeeman interaction term. It is shown that the resulting system has an underlying $\mathcal{N}=2$ supersymmetry provided Zeeman interaction contains the effective magnetic field instead of the external magnetic field. An alternative interpretation is that the Zeeman interaction still involves the external magnetic field, but, the Landé $g$-factor is modified in presence of loss-gain terms. The spectra and the state-space structure of the resulting Hamiltonian is identical with the standard Pauli Hamiltonian.

The plan of presentation of the results is the following. The formalism of the problem along with the general results are described in Sec. 2. The role of Lorentz interaction for having a semi-positive definite Hamiltonian is discussed in sub-section 2.1. The Hamiltonian formulation involves representation of some matrices which are presented in Sec. 2.2. The Sec. 2.3 contains discussions on an effective description of the system where loss-gain terms are absent and the system is subjected to an effective external magnetic field. The Landau Hamiltonian with balanced loss and gain is introduced in Sec. 3 with the results for the classical and the quantum systems in Sec. 3.1 and Sec. 3.2, respectively. The Hall effect in presence of balanced loss and gain is described in Sec. 3.3. In Sec. 3.4, Pauli equation with balanced loss and gain is shown to be supersymmetric. Finally, the results are summarized in Sec. 4. The Appendix-A in Sec. 5 contains representation of matrices for which balancing of loss-gain terms are not necessarily in a pair-wise fashion.

\section{Formalism \& General result}

Bateman's approach to find a Hamiltonian for a dissipative system is to embed it in an ambient space with twice the degrees of freedom of the original system 15. The extra degrees of freedom constitute an auxiliary system and the Hamiltonian is obtained for the combined original plus the auxiliary systems. In this approach, neither the original nor the auxiliary system alone is Hamiltonian. A Hamiltonian formulation for a system with both loss and gain without the introduction of an auxiliary system needs a separate treatment from that of Bateman. The point may be explained in terms of a system governed by the equations of motion:

$$
\ddot{x}_{i}+\sum_{k=1}^{N} \eta_{i k}\left(x_{1}, \ldots, x_{N}\right) \dot{x}_{k}+\Gamma_{i}\left(x_{1}, \ldots, x_{N}\right)=0, i=1, \ldots, N .
$$

The $i^{\text {th }}$ particle of the system is subjected to a velocity-independent force $-\Gamma_{i}$, gain/loss proportional to $\eta_{i i}$ and a velocity mediated coupling $\eta_{i k}(i \neq k)$ with the $k^{t h}$ particle. The particle is subjected to loss(gain) if $\eta_{i i}$ is positive(negative) at a point in the configuration space. The idea behind introducing an auxiliary system in Bateman's approach is to make the system nondissipative in the ambient space so that a Hamiltonian formulation is possible. The system governed by Eq. (1) contains both loss and gain, thereby, leading to the possibility that it may be non-dissipative under certain condition and without the introduction of an auxiliary system. This also raises the possibility of a Hamiltonian formulation of the system governed by Eq. (1) without the introduction of an ambient space or auxiliary system.

The condition for the system defined by Eq. (1) to be non-dissipative may be determined following the standard techniques in classical dynamics. In particular, the equations of motion (1) can be re-written as $2 N$ coupled first order differential equations in terms of two $2 N$ dimensional vectors $\vec{\xi}$ and $\vec{G}\left(\xi_{1}, \ldots, \xi_{2 N}\right)$ as $\dot{\vec{\xi}}=\vec{G}$, where

$$
\xi_{i} \equiv x_{i}, \xi_{N+i} \equiv \dot{x}_{i}, G_{i} \equiv \xi_{N+i}, G_{N+i} \equiv-\Gamma_{i}\left(\xi_{1}, \ldots, \xi_{N}\right)-\sum_{k=1}^{N} \eta_{i k}\left(\xi_{1}, \ldots, \xi_{N}\right) \xi_{N+k} .
$$


The criteria for a non-dissipative system is that the flow preserves volume in the $2 N$ dimensional position-velocity state space spanned by $\xi_{i}$ 's, which is equivalent to putting the condition that $\vec{G}$ is solenoidal, i.e. $\vec{\nabla}_{\xi} \cdot \vec{G}=0$. A straightforward calculation shows,

$$
\vec{\nabla}_{\xi} \cdot \vec{G} \equiv \sum_{i=1}^{2 N} \frac{\partial G_{i}}{\partial \xi_{i}}=\sum_{i=1}^{N} \eta_{i i}
$$

implying that the condition for a non-dissipative system is

$$
\sum_{i=1}^{N} \eta_{i i}=0
$$

The trivial solution $\eta_{i i}=0 \forall i$ is discarded from the ambit of further discussions, since it does not correspond to gain and/or loss for any individual particle. Eq. (44) is also the condition for the system governed by Eq. (1) to be identified as a system with balanced loss and gain. The reason is that the flow preserves the volume in the position-velocity state space spanned by $\xi_{i}$ 's, although individual particles are subjected to gain and/or loss.

One notable aspect of Bateman's formulation is that the dynamics of a dissipative linear or nonlinear system is completely decoupled from that of the dynamics of the corresponding auxiliary system [15, 5, 10. The distinction between original system and its auxiliary counterpart ceases to exists, if an interaction is added to the ambient space Hamiltonian such that their dynamics are intertwined to each other 8, 9, 10. This feature may be used to rule out the possibility that Eqs. (11) and (4) do not correspond to a combination of a hidden system plus its auxiliary part. In particular, the force $\Gamma_{i}$ on the $i^{t h}$ particle may always be chosen appropriately so that the dynamics of particles subjected to gain and that of particles with loss are intertwined to each other. This gives rise to a new class of system with balanced loss and gain that is different from the models obtained via Bateman's prescription. Further discussions in this paper are restricted to this distinct class of system with balanced loss and gain.

Is it possible to find a Hamiltonian for the system with balanced loss and gain that is governed by Eqs. (1) and (4) without introducing any auxiliary system? There is no definite answer for the most general case with arbitrary $\Gamma_{i}$ and $\eta_{i k}$. Even for a simpler case of $\eta_{i k}=(-1)^{i+1} \delta_{i k}, \Gamma_{i}=\frac{\partial \Gamma}{\partial x_{i}}$ with even $N$ and $\Gamma$ being the potential of rational Calogero model, no definite answer is known for $N>2$ [8]. The Hamiltonian formulation of systems with balanced loss and gain [8, 10, which is summarized below, constitute a special case of Eq. (11), albeit encompassing a very large class of such models. The importance of the formalism lies in the fact that no auxiliary system is introduced.

A Hamiltonian formulation of many-particle systems with space-dependent balanced loss and gain is presented in Refs. [8, 10. The analysis excludes constrained systems, systems with the dissipative term depending nonlinearly on the velocity and any other non-standard Hamiltonian formulations. The Hamiltonian is written as,

$$
H=\Pi^{T} \mathcal{M} \Pi+V\left(x_{1}, x_{2}, \ldots x_{N}\right),
$$

where $\mathcal{M}$ is a $N \times N$ real symmetric matrix with $X=\left(x_{1}, x_{2}, \ldots x_{N}\right)^{T}$ and $\Pi=\left(\pi_{1}, \pi_{2}, \ldots \pi_{N}\right)^{T}$ are $N$ coordinates and generalized momenta, respectively. The suffix $T$ in $O^{T}$ denotes the transpose of a matrix $O$. The matrix $\mathcal{M}$ may be interpreted as a constant background metric. This is evident if the first term of $H$ is expressed in terms of the generalized momenta $\pi_{i}$ as,

$$
\Pi^{T} \mathcal{M} \Pi=\sum_{i, k=1}^{N} \pi_{i} \mathcal{M}_{i k} \pi_{k} .
$$


The matrix $\mathcal{M}$ is non-singular and $\mathcal{M}^{-1}$ exists. However, $\mathcal{M}$ is not necessarily semi-positive definite. It may be noted in this context that the Hamiltonian of the Bateman oscillator [15] may also be identified as defined in the background of a pseudo-Euclidean metric with the signature $(1,-1)$ [16. Moreover, all the Hamiltonian systems with balanced loss and gain considered in the literature 1, 2, 3, 4, 5, 6, 7, prior to the general formulation of such systems in Ref. [8, 9, 10, may be identified as defined in the background of a pseudo-Euclidean metric.

The generalized momenta $\Pi$ is defined by,

$$
\Pi=P+A F(X)
$$

where $P=\left(p_{1}, p_{2}, \ldots, p_{N}\right)^{T}$ is the conjugate momentum corresponding to the coordinate $X$, $F(X)=\left(F_{1}, F_{2}, \ldots F_{N}\right)^{T}$ is $N$ dimensional column matrix whose entries are functions of coordinates and $A$ is an $N \times N$ anti-symmetric matrix. The reason for considering $A$ to be antisymmetric is that the symmetric part of a general matrix replacing $A$ will not contribute to the equations of motion resulting from $H[8$. Further, its contribution to the quantum mechanical wave-function can always be removed via a gauge transformation. 8]. The generalized momenta $\Pi$, when written in component form, has the following expression:

$$
\pi_{i}=p_{i}+a_{i}, \quad a_{i} \equiv \sum_{k=1}^{N} A_{i k} F_{k}
$$

where $a_{i}$ can be interpreted as components of a vector gauge potential $\vec{a}$. The introduction of the matrix $A$ to define the gauge potential $\vec{a}$ instead of the vector field $F$ alone, is solely a matter of convenience for formulating the problem in terms of arbitrary $F$. In general, $\vec{a}$ consists of two parts, $\vec{a}=\vec{a}_{r}+\vec{a}_{f}$. The vector $\vec{a}_{r}$ leads to realistic external magnetic field. If there is no external magnetic field in the system, $\vec{a}_{r}$ may be taken to be zero. The vector potential $\vec{a}_{f}$ is introduced to get the effect of loss/gain terms. In fact, the magnitude of the analogous magnetic field corresponding to $\vec{a}_{f}$ is identical with the loss/gain co-efficients [8, 10. This interpretation is valid for Bateman oscillator as well as all other examples considered so far.

The Lagrangian corresponding to the Hamiltonian $H$ in Eq. (5) has the following form:

$$
\mathcal{L}=\frac{1}{4} \dot{X}^{T} \mathcal{M}^{-1} \dot{X}-\frac{1}{2}\left(\dot{X}^{T} A F+F^{T} A^{T} \dot{X}\right)-V\left(x_{1}, x_{2}, \ldots, x_{N}\right) .
$$

The equations of motion derived from the Lagrangian (9) or the Hamiltonian (5) with the generalized momenta $\Pi$ defined by Eq. (7) read,

$$
\ddot{X}-2 \mathcal{M} R \dot{X}+2 \mathcal{M} \frac{\partial V}{\partial X}=0
$$

where the anti-symmetric matrix $R$ and $\frac{\partial V}{\partial X}$ are defined as follows:

$$
R \equiv A J-(A J)^{T}, \quad[J]_{i j} \equiv \frac{\partial F_{i}}{\partial x_{j}}, \quad \frac{\partial V}{\partial X} \equiv\left(\frac{\partial V}{\partial x_{i}}, \frac{\partial V}{\partial x_{2}}, \ldots \frac{\partial V}{\partial x_{N}}\right)^{T}
$$

It may be noted that the anti-symmetric nature of $R$ follows from its definition and is not imposed. Defining a matrix $\mathcal{D}$ as,

$$
\mathcal{D} \equiv \mathcal{M} R
$$

and comparing Eqs. (10) with (1), one to one correspondence may be made between these two equations through the following identifications:

$$
\eta=-2 \mathcal{D}, \quad \Gamma_{i}=2 \sum_{k=1}^{N} M_{i k} \frac{\partial V}{\partial x_{k}}
$$


It immediately follows from Eq. (4) that the condition for a balanced loss-gain system is $\operatorname{Tr}(\mathcal{D})=$ 0 . The symmetric and anti-symmetric nature of the matrices $\mathcal{M}$ and $R$, respectively, ensures that the condition $\operatorname{Tr}(\mathcal{D})=\sum_{i=1}^{N} \mathcal{M}_{i k} R_{k i}=0$ indeed holds for the Hamiltonian $H$. The Hamiltonian $H$ is identified as describing a system with equally balanced loss and gain, since individual particles are subjected to gain or loss such that net gain or loss of energy is zero.

\subsection{Lorentz interaction \& a semi-positive definite $\mathcal{M}$}

In Refs. [8, 10, it is shown on general ground that for any symmetric matrix $\mathcal{D}$,

$$
\{\mathcal{M}, R\}=0,\{\mathcal{M}, \mathcal{D}\}=0,\{R, \mathcal{D}\}=0 .
$$

This implies that the eigenvalues of the $2 m \times 2 m$ matrix $\mathcal{M}$ are $\left(\lambda_{1}, \ldots, \lambda_{m},-\lambda_{1}, \ldots,-\lambda_{m}\right)$, while for odd-dimensional $\mathcal{M}$ an extra eigenvalue 1 is added to it. Thus, the matrix $\mathcal{M}$ is not semi-positive definite for a symmetric $\mathcal{D}$. Stable classical solutions are possible for specific choices of the potentials and parameter ranges. However, except for one known case involving a quasi-exactly solvable model with anaharmonic interaction[10, quantum bound states exist for such systems only if the eigenvalue problem is extended to complex domain. In particular, normalizable eigenfunctions for bound states are obtained in specific Stoke wedges on the complex plane. Although the complex extension of quantum mechanics is mathematically consistent, no experimental verification of this has been found so far. One of the motivations of this article is to present a model independent formulation of Hamiltonian system with balanced loss and gain so that the quantum problem is well-defined on the real line. The non-normalizability of eigenfunctions on the real line may be traced back to the non-positivity of $\mathcal{M}$ which is a consequence of a symmetric $\mathcal{D}$ through the relations in Eq. (14).

Investigations [8, 10, 9] on Hamiltonian systems with balanced loss and gain have been so far restricted to a diagonal $\mathcal{D}$ which is a symmetric matrix. This is primarily because only the diagonal elements of $\mathcal{D}$ are relevant for determining whether a system is dissipative or nondissipative. Nevertheless, the off-diagonal elements of $\mathcal{D}$ give rise to a variety of interesting physical effects. For example, the equation of motion for a particle subjected to Lorentz force in appropriate units is given by,

$$
\ddot{x}_{i}=E_{i}+\epsilon_{i j k} \dot{x}_{j} B_{k}, i=1,2,3,
$$

where $E_{i} \equiv E_{i}\left(x_{1}, x_{2}, x_{3}\right)$ and $B_{i} \equiv B_{i}\left(x_{1}, x_{2}, x_{3}\right)$ are components of electric and magnetic fields. Eq. (15), when cast into the form of Eq. (10), leads to an anti-symmetric $\mathcal{D}$ with its elements $\mathcal{D}_{i j}=-\frac{1}{2} \epsilon_{i j k} B_{k}$. Further, when the equations governing synchronization of velocity-coupled systems are cast into the form (10), the matrix $\mathcal{D}$ contains diagonal as well as off-diagonal elements. For example, the non-vanishing diagonal as well as off-diagonal elements of $\mathcal{D}$ appear in the study of stochastic synchronization of oscillation in systems of velocity-coupled oscillators with individual chaotic dynamics 17] for which off-diagonal part of $\mathcal{D}$ is symmetric. Similarly, in the case of synchronization of velocity-coupled limit-cycle oscillators, the off-diagonal part of $\mathcal{D}$ is symmetric 18,19 . The trace of $\mathcal{D}$ is non-vanishing for both the cases and the systems are dissipative. Similar velocity dependent coupling also arises in the description of partially ionized plasma 20]. Thus, the domain of applicability of Hamiltonian systems with balanced loss and gain may be enlarged by considering off-diagonal elements of $\mathcal{D}$.

A symmetric $\mathcal{D}$ leads to negative eigenvalues of $\mathcal{M}$. Although $\mathcal{M}$ may be chosen to be semi-positive definite for an anti-symmetric $\mathcal{D}$, no gain and/or loss term can be incorporated in the system due to $\mathcal{D}_{i i}=0, \forall i$. Thus, the matrix $\mathcal{D}$ can neither be symmetric nor antisymmetric in order to describe a Hamiltonian system with balanced loss and gain such that the 
symmetric matrix $\mathcal{M}$ is semi-positive definite. In general, $\mathcal{D}$ may always be decomposed as the sum of a diagonal matrix $D$, a symmetric matrix with vanishing diagonal elements $D_{O}$ and an antisymmetric matrix $\mathcal{D}_{A}$ :

$$
\mathcal{D}=D+D_{O}+\mathcal{D}_{A}
$$

It may be noted that $\mathcal{D}_{S}=D+D_{O}$ is a symmetric matrix and $\operatorname{Tr}(\mathcal{D})=0$ implies $\operatorname{Tr}(D)=0$. The elements of $D$ are related to the loss/gain co-efficients, while elements of $\mathcal{D}_{A}$ are related to the Lorentz interaction. The elements of $D_{O}$ give rise to coupling among different particles through their velocities, which is different from Lorentz interaction and appear in the study of many systems including synchronization of different types of oscillators [18, 19] and in the description of partially ionized plasma 20]. All three matrices correspond to interesting physical situations. It may be possible to describe a Hamiltonian system with balanced loss and gain so that $\mathcal{M}$ is positive definite, even if $D_{O}$ is taken to be a zero matrix. However, this is impossible if either $D$ or $\mathcal{D}_{A}$ is taken to be a zero matrix. One very important consequence of a non-vanishing $\mathcal{D}_{A}$ is that balancing of gain/loss terms does not necessarily occur in a pair-wise fashion, unlike the previous cases $[8,9,10$. The balancing of loss/gain terms are possibles in as many ways as the solutions of $\operatorname{Tr}(\mathcal{D})=0$ can be realized. For example, $N_{g}$ particles may be subjected to gain with coefficients $g_{i}$ and $N_{l}$ particles may be be subjected to loss with coefficients $l_{i}$, such that $\sum_{i=1}^{N_{g}} g_{i}-\sum_{i=1}^{N_{l}} l_{i}=0, N=N_{g}+N_{l}$. The case of pair-wise balancing of loss/gain terms appears as a special case. Such a formulation allows more flexibility in constructing and controlling Hamiltonian systems with balanced loss and gain.

\subsection{Representation of matrices}

The matrix $\mathcal{D}$ can be decomposed as the product of a symmetric and an anti-symmetric matrix if it is similar to $-\mathcal{D}[21$. However, the representation of the matrices $\mathcal{M}, R$ and $\mathcal{D}$ satisfying Eq. (12) is not unique and various choices may be made depending on physical situations. It may be noted that a diagonal $\mathcal{M}$ necessarily leads to a $\mathcal{D}$ with all of its diagonal elements to be zero and can not describe a system with balanced loss and gain. On the other hand, a traceless $\mathcal{M}$ is not semi-positive definite. Thus, the necessary conditions for the choice of $\mathcal{M}$ is the following:

1. $\operatorname{Trace}(\mathcal{M}) \neq 0$

2. $[\mathcal{M}]_{i j} \neq 0, i \neq j$ for one or more pairs of $(i, j)$

The sufficient condition for $\mathcal{M}$ to be positive-definite is to be checked separately. The product of any positive definite $\mathcal{M}$ with non-vanishing off-diagonal elements and an anti-symmetric matrix $R$ will determine the matrix $\mathcal{D}$. The matrix $\mathcal{D}$ can always be decomposed as in Eq. (16):

$$
\mathcal{D}_{S}=\frac{1}{2}[\mathcal{M}, R], \quad \mathcal{D}_{A}=\frac{1}{2}\{\mathcal{M}, R\} .
$$

A few representations of the matrices are presented in Appendix-A for arbitrary $N$, where the balancing of loss-gain terms does not necessarily occurs in a pair-wise fashion. If the off-diagonal elements of $\mathcal{D}$ are to be related to contributions coming from Lorentz interaction only, then $\mathcal{D}_{O}$ must be taken to be zero and $\mathcal{D}$ is decomposed as,

$$
\mathcal{D} \equiv D+\mathcal{D}_{A}
$$

A particular representation of Eqs. (12) and (18) for $N=2 m$ with pair-wise balancing of loss/gain terms is considered here to show that $\mathcal{M}$ can indeed be chosen to be positive definite. The matrix $\mathcal{M}$ is chosen as,

$$
\mathcal{M}=M+\alpha^{2} I_{2 m}
$$


where $M$ is a traceless $2 m \times 2 m$ symmetric matrix, $I_{2 m}$ is the $2 m \times 2 m$ identity matrix and $\alpha$ is a real parameter. Note that both $\mathcal{M}$ and $M$ are simultaneously diagonalizable. Substituting $\mathcal{M}$ in Eq. (12), the decomposition of $\mathcal{D}$ as in Eq. (16) is obtained with the identification,

$$
D=M R, \mathcal{D}_{A}=\alpha^{2} R \text {. }
$$

The first equation of Eq. (20) involving symmetric matrices $M, D$ and anti-symmetric matrix $R$ implies that they anti-commute with each other. Thus, the eigenvalues of $\mathcal{M}$ are $\alpha^{2} \pm \lambda_{i}, i=$ $1, \ldots m$. The condition for semi-positive definite $\mathcal{M}$ is, $\alpha^{2} \geq \max _{i} \lambda_{i}$. Matrix representation of the first equation of Eq. (20) is suffice to completely specify the representation of $\mathcal{M}, \mathcal{D}$ and $R$. A few representations of the equation $D=M R$ have been discussed in Ref. [8] for systems with constant balanced loss-gain terms. For the case of space-dependent balanced loss-gain terms with $F_{i} \equiv F_{i}\left(x_{2 i-1}, x_{2 i}\right)$, a particular representation of the matrices is presented in Ref. [10], which is reproduced below for further discussions:

$$
M=I_{m} \otimes \sigma_{x}, \quad A=\frac{-i \gamma}{2} I_{m} \otimes \sigma_{y}, \quad D=\gamma \chi_{m} \otimes \sigma_{z}, \quad\left[\chi_{m}\right]_{i j}=\frac{1}{2} \delta_{i j} Q_{i}\left(x_{2 i-1}, x_{2 i}\right),
$$

where $\sigma_{x}, \sigma_{y}, \sigma_{z}$ are Pauli matrices and $I_{m}$ is $m \times m$ identity matrix. The $m$ functions $Q_{i}$ appearing in the $m \times m$ diagonal matrix $\chi_{m}$ is determined as,

$$
Q_{a}\left(x_{2 a-1}, x_{2 a}\right)=\operatorname{Trace}\left(V_{a}^{(2)}\right), \quad V_{a}^{(2)} \equiv\left(\begin{array}{ll}
\frac{\partial F_{2 a-1}}{\partial x_{2 a-1}} & \frac{\partial F_{2 a-1}}{\partial x_{2 a}} \\
\frac{\partial F_{2 a}}{\partial x_{2 a-1}} & \frac{\partial F_{2 a}}{\partial x_{2 a}}
\end{array}\right)
$$

It may be noted that the matrix $J$ has a block-diagonal form for the choices of $F_{i} \equiv F_{i}\left(X_{2 i-1}, x_{2 i}\right)$ and $R$ may be determined from the first two equations of Eq. (11) by using the expressions of $A$ and $J$ :

$$
R=\frac{\gamma}{2} \sum_{i=1}^{m} U_{i}^{(m)} \otimes\left(\begin{array}{cc}
0 & -Q_{i}\left(x_{2 i-1}, x_{2 i}\right) \\
Q_{i}\left(x_{2 i-1}, x_{2 i}\right) & 0
\end{array}\right), \quad\left[U_{a}^{(m)}\right]_{i j}=\delta_{i a} \delta_{j a},
$$

where $U_{a}^{(m)}$ are $m$ numbers of $m \times m$ matrices. The eigenvalues of $M$ are \pm 1 . Thus, $\mathcal{M}$ is positive-definite for $\alpha^{2}>1$. This completely specifies the representation of $\mathcal{M}, R$ and $\mathcal{D}$. This representation for even number of particles can be generalized 8 to $N=2 m+1$ such that the dynamics of $x_{2 m+1}$ does not contain any gain/loss term and interacts with all other particles through the interaction potential. This can be achieved by adding an extra column and a row to $\mathcal{M}, R, \mathcal{D}$ with all vanishing elements except for $\mathcal{M}_{2 m+1,2 m+1}$. The case of constant balanced loss and gain may be reproduced $\left[\underline{8}\right.$ for $F_{2 i-1}=x_{2 i-1}$ and $F_{2 i}=x_{2 i}$.

The representation specified by Eqs. (21, 22, 23) determines $\mathcal{D}$ with $\mathcal{D}_{O}$ being a null matrix. However, the same representations with a modified $\mathcal{M}$,

$$
\mathcal{M}=\beta_{1} M+\alpha^{2} I_{2 m}+\beta_{2} I_{m} \otimes \sigma_{z}, \beta_{1}, \beta_{2} \in \Re,
$$

may be used to get the following expression of $\mathcal{D}=\beta_{1} D+\beta_{2} D_{O}+\alpha^{2} R$ with $D_{O}$ having nonvanishing elements:

$$
D_{O}=\frac{\beta_{2} \gamma}{2} \sum_{i=1}^{m} U_{i}^{(m)} \otimes\left(\begin{array}{cc}
0 & -Q_{i}\left(x_{2 i-1}, x_{2 i}\right) \\
-Q_{i}\left(x_{2 i-1}, x_{2 i}\right) & 0
\end{array}\right) .
$$

The eigenvalues of $\mathcal{M}$ are $\alpha^{2} \pm \sqrt{\beta_{1}^{2}+\beta_{2}^{2}}$ with multiplicity $m$ for each eigenvalue. The matrix $\mathcal{M}$ is positive definite for $\alpha^{2}>\sqrt{\beta_{1}^{2}+\beta_{2}^{2}}$. 


\subsection{Hiding the loss-gain terms}

It is known 8, 9, 10 that Hamiltonian systems with balanced loss-gain and without any external magnetic field can always be interpreted as defined in the background of a pseudo-Euclidean metric and subjected to external 'analogous magnetic field' having the same spatial form as the gain-loss co-efficient. The equations of motion in the new co-ordinate system do not contain any gain-loss terms. A similar investigation for the case of a realistic external magnetic field is performed in this section. The matrix $\mathcal{M}$ being a real symmetric matrix, it can be diagonalized by an orthogonal matrix $\hat{O}$, i.e. $M_{d}=\hat{O}^{T} \mathcal{M} \hat{O}$. The matrix $M_{d}$ and two other matrices $S$ and $\eta^{a}$ are defined as follows:

$$
\left[M_{d}\right]_{i j}=\epsilon_{i} \delta_{i j}\left|\lambda_{i}\right|, \quad[S]_{i j}=\delta_{i j} \sqrt{\left|\lambda_{i}\right|}, \quad\left[\eta^{a}\right]_{i j}=\delta_{i j} \epsilon_{i},
$$

where $\lambda_{i}$ 's are the eigenvalues of the matrix $\mathcal{M}$ and $\epsilon_{i}$ 's take value of 1 or -1 . It may be noted that $\epsilon_{i}$ 's keep track of whether a particular eigenvalue $\lambda_{i}$ is positive or negative. The parameter space of the system can be divided into at most $N+1$ regions depending on the number of positive eigenvalues of the matrix $\mathcal{M}$. For example, the region with $N$ positive eigenvalues may be denoted as Region-I, the region with $N-1$ positive eigenvalues may be denoted as RegionII and so on with the region with no positive eigenvalues is denoted as $\operatorname{Region}-(N+1)$. The superscript of $\eta^{a}$ labels each region and for a fixed ' $a$ ', $\eta^{a}$ is valid in that particular region only. For example, $\eta^{I}=I_{N}$ in Region-I, while $\eta^{N+1}=-I_{N}$ in $\operatorname{Region-}(N+1)$. The matrix $\eta^{a}$ is to be interpreted as the background metric for an effective description of the system defined by the Hamiltonian $H$ and equations of motion following from it in Eqs. (510).

The system described by the equations of motion (10) is non-dissipative, although individual particles are subjected to gain or loss. Are the appearance of gain-loss terms an artifact of choice of the co-ordinate system and can be removed completely in an another frame? In order to answer this question, a new co-ordinate system $[8$ is defined as follows:

$$
\mathcal{X}=S^{-1} \hat{O}^{T} X, \quad \mathcal{P}=S \hat{O}^{T} P,
$$

where $\mathcal{X} \equiv\left(\mathcal{X}_{1}, \mathcal{X}_{2}, \ldots, \mathcal{X}_{N}\right)^{T}$ and $\mathcal{P} \equiv\left(\mathcal{P}_{1}, \mathcal{P}_{2}, \ldots, \mathcal{P}_{N}\right)^{T}$. The matrix $S$ along with its inverse $S^{-1}$ is used to generate canonical scale transformation for the rotated co-ordinates $\hat{X} \equiv \hat{O}^{T} X$ and the rotated momenta $\hat{P} \equiv \hat{O}^{T} P$, where $\hat{X} \equiv\left(\hat{X}_{1}, \hat{X}_{2}, \ldots \hat{X}_{N}\right)^{T}$ and $\hat{P} \equiv\left(\hat{P}_{1}, \hat{P}_{2}, \ldots \hat{P}_{N}\right)^{T}$. The kinetic energy term of $H$ is in diagonal form in the rotated co-ordinate system $\hat{X}, \hat{P}$. The scale transformation is performed so that all the coefficients of the $\hat{P}_{i}^{2}$ terms are normalized to unity. Thus, the background metric in the co-ordinate system by $\mathcal{X}$ may be identified as (pseudo-)Euclidean one. The matrices $\mathcal{M}, R$ and $\mathcal{D}$ are transformed as follows:

$$
\begin{aligned}
& S^{-1} \hat{O}^{T} \mathcal{M} \hat{O} S^{-1}=\eta^{a}, \\
& {[\mathcal{R}]_{i j}=\left[O^{T} R O\right]_{i j} \sqrt{\left|\lambda_{i}\right|\left|\lambda_{j}\right|},} \\
& S^{-1} O^{T} \mathcal{D} O S=\eta^{a} \mathcal{R},
\end{aligned}
$$

which are obtained by simply using the rules of matrix multiplication. It may be noted that $\mathcal{R}$ is anti-symmetric, since $R^{T}=-R$. The first and the third equations in Eq. (28) are different in different regions, while the second equation has the same expression in all the regions. It may be noted that $S^{-1}$ and $S$ are not generating any similarity transformation for $\hat{O}^{T} \mathcal{M O}$ and $\hat{O}^{T} R \hat{O}$, respectively. Consequently, eigenvalues of $\mathcal{M}$ and $R$ are modified. However, $O S$ indeed generates a similarity transformation for $\mathcal{D}$ and the eigenvalues of $\mathcal{D}$ remain unchanged.

The generalized momenta $\Pi$ transforms like $P$ and denoted as $\hat{\Pi}$ in the new co-ordinates:

$$
\hat{\Pi} \equiv S \hat{O}^{T} \Pi=\mathcal{P}+\frac{\mathcal{R}}{2} \mathcal{X}
$$


which may be obtained by using the canonical transformation in Eq. (27). The Hamiltonian and the equations of motion resulting from it in different regions have the forms:

$$
\begin{aligned}
& H^{a}=\hat{\Pi}^{T} \eta^{a} \hat{\Pi}+\mathcal{V}\left(\mathcal{X}_{1}, \mathcal{X}_{2}, \ldots, \mathcal{X}_{N}\right) \\
& \ddot{\mathcal{X}}-2 \eta^{a} \mathcal{R} \dot{\mathcal{X}}+2 \eta^{a}\left(\frac{\partial \mathcal{V}}{\partial \mathcal{X}}\right)=0, \quad \mathcal{V}\left(\mathcal{X}_{1}, \mathcal{X}_{2}, \ldots, \mathcal{X}_{N}\right)=V\left(x_{1}, x_{2}, \ldots, x_{N}\right) .
\end{aligned}
$$

There are no loss and/or gain terms in the equations of motion (30) in terms of $\mathcal{X}$, since $\left(\eta^{a} \mathcal{R}\right)_{i i}=0 \forall i, a$. The effect of removing the gain-loss terms from Eq. (10) is to modify the coefficients of the velocity-mediated non-Lorentzian interaction and/or the magnitude of the magnetic field, since $\mathcal{D}_{i j} \neq\left(\eta^{a} R\right)_{i j}, \forall i, j$. In particular, the velocity dependent non-Lorentzian interaction vanishes in region-I and region- $(\mathrm{N}+1)$ in the effective description, since $\eta^{a} R$ becomes anti-symmetric. The applied magnetic field is modified to an effective magnetic field whose components are related to the elements of the $\eta^{a} \mathcal{R}$. In Region-II to Region- $N$, both non-Lorentzian and Lorentzian interactions are present with modified coupling co-efficients, since $\eta^{a} \mathcal{R}$ is neither symmetric nor anti-symmetric. However, with specific representations of $\mathcal{M}$ and $R, \eta^{a} R$ may be made to be symmetric or anti-symmetric.

A few advantages of using Eq. (30) over Eqs. (5) and (10) in analyzing the system may be mentioned. In Region-I and Region- $(\mathrm{N}+1)$, the quantum problem may be defined on the real line instead of Stokes wedges on the complex plane. There is a vast literature on systems in presence of external magnetic field, which may be used efficiently to study the classical as well as quantum system. In Region-II to Region-N, use of imaginary scaling of the co-ordinates 12 . associated with negative signatures may be helpful to analyze the quantum Hamiltonian. As far as the classical system is concerned, which form is to be used for finding the solution is a matter of convenience. However, it seems by analyzing a number of problems that Eq. (30) is relatively simpler to solve than Eq. (10).

\section{Landau Hamiltonian with balanced loss and gain}

It has been shown that that an external magnetic field allows the matrix $\mathcal{M}$ to be positive-definite and thereby, raising the possibility of improved stability properties of the system. A system subjected to (non-)uniform magnetic field appears in many areas of physics. The examples include Zeeman effect, Cyclotron, Hall effect, spintronics, neutron stars, plasma, etc. Thus, it is pertinent to consider systems where magnetic field is essentially required to describe a physical phenomenon and generalize them by including balanced loss-gain terms. The celebrated Landau Hamiltonian with balanced loss-gain is considered in this section at the classical as well quantum level.

\subsection{Classical system}

A two dimensional system with uniform balanced loss-gain terms is considered. Thus, the functions $F_{i}$ are chosen as $F_{i}=x_{i}$ and $N=2$. The condition of constant loss-gain terms imposed by the choice of $F_{i}$ also implies that the matrix $J$ is an identity matrix and hence, $A=\frac{1}{2} R$. The representation of the matrices $\mathcal{M}, R, \mathcal{D}$ may be considered as follows:

$$
\mathcal{M}=\frac{1}{2}\left(\begin{array}{cc}
B+C & \gamma \\
\gamma & B-C
\end{array}\right), R=\left(\begin{array}{cc}
0 & 1 \\
-1 & 0
\end{array}\right), \mathcal{D}=\frac{1}{2}\left(\begin{array}{cc}
-\gamma & B+C \\
-(B-C) & \gamma .
\end{array}\right)
$$

Decomposing $\mathcal{D}$ as in Eq. (16), it is apparent from Eq. (10) that the particle is subjected to a uniform magnetic field $B$ along the perpendicular to the ' $x_{1}-x_{2}$ '-plane. A change in the direction 
of the magnetic field is accomplished by taking $B \rightarrow-B$. The vanishing field $B=0$ corresponds to $\operatorname{Trace}(\mathcal{M})=0$ and $\mathcal{M}$ is not positive-definite. Apart from the Lorentz interaction 1 , a velocity mediated coupling between the two degrees of freedom with the strength $C$ is also present in the system. The discussions in this article will be based on generic $B$ and $C$ unless specified otherwise. The case of pure Lorentz interaction may be discussed by employing the limit $C=0$. The particle is subjected to balanced loss and gain with the strength $\gamma$. The eigenvalues of the matrix $\mathcal{M}$ are,

$$
\lambda_{ \pm}=\frac{1}{2}(B \pm \triangle), \triangle \equiv \sqrt{C^{2}+\gamma^{2}} .
$$

The matrix $\mathcal{M}$ becomes singular for $B= \pm \triangle$ and these two conditions determine the boundaries of three different regions in the parameter space of $B$ and $\triangle$ :

- Region-I $(B>\triangle)$ ): The matrix $\mathcal{M}$ is positive-definite. This condition for the case of pure Lorentz interaction(i.e. $C=0$ ) implies that the magnitude of the external magnetic field must be greater than the magnitude of the gain/loss co-efficient $\gamma$. It appears that a positive-definite $\mathcal{M}$ has not been considered earlier in the literature. Thus, this region is of special interest for the present article. The diagonal matrix $\eta^{I}=I_{2}$.

- Region-II $\left(-\triangle<B<\triangle\right.$ ): One of the eigenvalues $\lambda_{-}$is negative, while $\lambda_{+}$is positive. All previous studies [2, 8, 9, 10, on Hamiltonian systems with balanced loss/gain dealt with the case $\lambda_{+}=-\lambda_{-}$, which is contained in the present case for $B=0$. However, for $B \neq 0, \lambda_{+}$ and $-\lambda_{-}$are different. It is expected that for $B \neq 0$ there may exist sub-regions within this region in which the classical system admits periodic solution and the corresponding quantum theory admits well defined bound states in specific Stoke wedges as in the case for $B=0$. The diagonal matrix $\eta^{I I}=\sigma_{z}$

- Region-III $(B<-\triangle)$ : Both the eigenvalues $\lambda_{ \pm}$are negative and it appears that such a situation has not been considered earlier in the investigations on Hamiltonian system with balanced loss/gain. The Hamiltonian is not bounded from below, but, bounded from above. An inclusion of an appropriate potential $V$ in the system may allow the Hamiltonian to be bounded from below, thereby, raising the possibility of classical as well as quantum bound states. The diagonal matrix $\eta^{I I I}=-I_{2}$.

The orthogonal matrix $\hat{O}$ that diagonalizes $\mathcal{M}$ to $M_{d}=\hat{O}^{T} \mathcal{M} \hat{O}$ has the form:

$$
M_{d}=\left(\begin{array}{cc}
\lambda_{+} & 0 \\
0 & \lambda_{-}
\end{array}\right), \quad \hat{O}=\frac{1}{\sqrt{\gamma^{2}+(\triangle-C)^{2}}}\left(\begin{array}{cc}
\gamma & -(\triangle-C) \\
\triangle-C & \gamma
\end{array}\right) .
$$

The matrix $\mathcal{M}$ is diagonal for $\gamma=0$ for which $\hat{O}$ is not defined. The diagonal matrix $S$ has the non-vanishing elements, $[S]_{11}=\sqrt{\left|\lambda_{+}\right|}$and $[S]_{22}=\sqrt{\left|\lambda_{-}\right|}$. The transformations (27), with $\hat{O}$ and $S$ as given above, consist of a rotation in the ' $x_{1}-x_{2}$ '-plane by an angle $\theta=\tan ^{-1}\left(\frac{\Delta-C}{\gamma}\right)$ followed by scaling of $\hat{X}_{1}$ and $\hat{X}_{2}$ by $\frac{1}{\sqrt{\left|\lambda_{+}\right|}}$and $\frac{1}{\sqrt{\left|\lambda_{-}\right|}}$, respectively. The canonical transformation is not defined at the two boundaries $(B= \pm \triangle)$ of the three regions and within each region for $\gamma=0$ and $C \neq 0$. Thus, the limit $\gamma=0$ is singular for $C \neq 0$. The results for standard Landau Hamiltonian can be reproduced from the results in the transformed co-ordinates $\left(\mathcal{X}_{1}, \mathcal{X}_{2}\right)$ by taking $C=0$ and then imposing the limit $\gamma \rightarrow 0$. The angle $\theta=\frac{\pi}{4}$ for $C=0, \gamma \neq 0$, which corresponds to velocity-dependent force due to Lorentz interaction only.

\footnotetext{
${ }^{1}$ An appropriate choice of $V$ produces an external electric field.
} 
The generalized $\hat{\Pi}$ in the new co-ordinates has the form:

$$
\hat{\Pi}=\mathcal{P}+\frac{|\omega|}{4} R \mathcal{X}, \omega \equiv \sqrt{B^{2}-\triangle^{2}} .
$$

The Hamiltonian and the equations of motion in this new co-ordinate system have the following expressions:

$$
H^{a}=\hat{\Pi}^{T} \eta^{a} \hat{\Pi}, \quad \ddot{\mathcal{X}}-|\omega| \eta^{a} R \dot{\mathcal{X}}=0 .
$$

It may be noted that there are no gain-loss terms in this new co-ordinate system, since the diagonal elements of $\eta^{a} R$ are zero. The absence of the gain-loss terms is compensated by the appearance of an effective magnetic field with its magnitude receiving contributions from the realistic external magnetic field and an analogous magnetic field. The Hamiltonian and the equations of motion in Eq. (35) correspond to the standard Landau problem for $\eta^{a}=I_{2}$ for which the centre of the cyclotron motion is a constant of motion. The same analysis may be generalized in a straightforward way to other allowed forms of $\eta^{a}$ to find corresponding constants of motion. The components of the vector $C^{a}=\left(C_{1}^{a}, C_{2}^{a}\right)^{T}$ are two constants of motion of the system:

$$
C^{a}=\mathcal{X}+\frac{1}{|\omega|} R \eta^{a} \dot{\mathcal{X}}=\hat{O} S\left(X+\frac{1}{|\omega|} R \eta^{a} \dot{X}\right) .
$$

The first expression on the right side of $C^{a}$ is the centre of cyclotron motion for the system defined by Eq. (35), which is re-written in terms of the original variables $X$ in the second expression by using Eq. (27). It may be checked that $\frac{d C^{a}}{d t}=0$ by using the equations of motion and the identities $R^{2}=-I,\left(\eta^{a}\right)^{2}=I, \forall a$. It may be noted that two independent constants of motion may be chosen for the system, depending on the physical requirements, by taking appropriate combinations of $C_{1}^{a}$ and $C_{2}^{a}$. The reason for the particular choice of $C^{a}$ is that it may be identified as the center of the cyclotron motion for $\eta^{a}=I_{2}$. Two complex parameters $\xi_{1}, \xi_{2}$ along with their polar decompositions are introduced as follows,

$$
\begin{aligned}
& \xi_{1}=\frac{2}{\sqrt{|\omega|}}\left(\sqrt{\left|\lambda_{+}\right|} \cos \theta+i \sqrt{\left|\lambda_{-}\right|} \sin \theta\right)=\left|\xi_{1}\right| e^{i \phi_{1}}, \\
& \xi_{2}=\frac{2}{\sqrt{|\omega|}}\left(-\sqrt{\left|\lambda_{+}\right|} \sin \theta+i \sqrt{\left|\lambda_{-}\right|} \cos \theta\right)=\left|\xi_{2}\right| e^{i\left(\phi_{2}+\frac{\pi}{2}\right)}, \\
& \phi_{1}=\tan ^{-1}\left(\sqrt{\frac{\left|\lambda_{-}\right|}{\left|\lambda_{+}\right|}} \tan \theta\right), \quad \phi_{2}=\tan ^{-1}\left(\sqrt{\frac{\left|\lambda_{+}\right|}{\left|\lambda_{-}\right|}} \tan \theta\right),
\end{aligned}
$$

which will be used for presenting the results in terms of the original co-ordinates $\left(x_{1}, x_{2}\right)$ in a compact form. It may be recalled that $C=0$ corresponds to the situation with Lorentz interaction only for which $\theta=\frac{\pi}{4}$ and $\xi_{1}=-\xi_{2}^{*}$. The nature of the solutions in three different regions are different and is described below separately. It is worth emphasizing here that no solutions at the two boundaries $B= \pm \triangle$ separating the three regions are presented. It may be noted at this point that the Hamiltonian formulation of balanced loss-gain system is based on the assumption of a non-singular $\mathcal{M}$, which is violated at these two boundaries. Thus, the discussions on solutions at the boundaries are beyond the purview of this article.

\subsubsection{Region-I}

The solutions may be written as,

$$
x_{1}=C_{1}^{I}+\frac{\left|\xi_{1}\right|}{2|\omega|}|Z| \cos \left(|\omega| t-\phi_{1}\right),
$$




$$
x_{2}=C_{2}^{I}-\frac{\left|\xi_{2}\right|}{2|\omega|}|Z| \sin \left(|\omega| t-\phi_{2}\right), \quad Z \equiv \xi_{1} \dot{x}_{2}+\xi_{2} \dot{x}_{1} .
$$

The quantity $\frac{|Z|}{|\omega|}$ is related to the cyclotron radius in the transformed co-ordinate $\left(\mathcal{X}_{1}, \mathcal{X}_{2}\right)$. The Hamiltonian $H$ in Region-I describes a system with balanced loss and gain for which its kinetic energy term is semi-positive definite. The system is interpreted as that of a particle moving on an Euclidean plane and subjected to an effective external magnetic field $|\omega|$, which is less than the applied magnetic field $B$. The role of the external magnetic field with a lower bound on its magnitude $B>\triangle$ is essential to achieve this. The effect of the inclusion of balanced loss-gain to the Landau Hamiltonian is to have a reduced value of the cyclotron frequency $|\omega|$ compared to its original magnitude $B$. The co-ordinate transformation induces a similarity transformation on the matrix $\mathcal{D}$, keeping its purely imaginary eigenvalues $\pm i \omega$ unchanged, the modulus of which determines the cyclotron frequency. Thus, the motion described in either of the co-ordinate systems has the same cyclotron frequency. However, the trajectories of the particle are circle in the co-ordinates $\left(\mathcal{X}_{1}, \mathcal{X}_{2}\right)$, while it is ellipse in the co-ordinates $\left(x_{1}, x_{2}\right)$. The unequal scaling of the two co-ordinates $\left(x_{1}, x_{2}\right)$ allows the elliptic orbits to be viewed as circular orbits in the co-ordinate system $\left(\mathcal{X}_{1}, \mathcal{X}_{2}\right)$. In general, for $C \neq 0, \gamma \neq 0$, the reduced value of the cyclotron frequency and an elliptic orbit is due to the effect of both the gain-loss coefficient $\gamma$ and the velocity mediated coupling $C$. However, this result is valid even if only Lorentz interaction is considered and the velocity mediated coupling $C=0$. In particular, in the limit of pure Lorentz interaction $(C=0)$, the co-efficient of the time-varying part for the two solutions are identical, since $\left|\xi_{1}\right|=\left|\xi_{2}\right|$ for $\theta=\frac{\pi}{4}$. However, the phases $\phi_{1}$ and $\phi_{2}$ are different.

\subsubsection{Region-II}

The solutions may be written as,

$$
X=C^{I I}+\frac{1}{2|\omega|}\left(Z^{2}+Z^{* 2}\right)^{\frac{1}{2}} \hat{O} S\left(\begin{array}{c}
\cosh |\omega| t \\
\sinh |\omega| t
\end{array}\right) .
$$

The kinetic energy term is not semi-positive definite. The Hamiltonian may be interpreted as that of a particle moving in the background of a pseudo-Euclidean metric that is subjected to an effective external magnetic field $|\omega|$. The magnitude of the external magnetic field is less than the magnitude of the analogous magnetic field. The known results for generic Hamiltonian of this form [8, 9, 10] for $B=C=0$ appears as a special case in this region with an 'analogous magnetic field' having the magnitude $\gamma$. For $B=0, C \neq 0$, the same interpretation is valid with the magnitude of the 'analogous magnetic field' being $\triangle$. However, the description changes for $B \neq 0$ for which the effective magnetic field receives contribution from both the applied as well as analogous magnetic field. The Hamiltonian $H^{I I}$ does not admit any periodic solution, as is evident from Eq. (39). The quantity $Z^{2}+Z^{* 2}$ is fixed by initial conditions and may be chosen to be positive so that $\left(Z^{2}+Z^{* 2}\right)^{\frac{1}{2}}$ is real. The solutions diverge in the limit of large $t$. The Hamiltonian for $B \neq 0, C \neq 0$ may admit periodic solutions if an appropriate non-vanishing $\mathcal{V}$ is added to the system. Such an investigation is beyond the scope of the present article.

\subsubsection{Region-III}

The solutions are,

$$
x_{1}=C_{1}^{I I I}+\frac{\left|\xi_{1}\right|}{2|\omega|}|Z| \cos \left(|\omega| t-\phi_{1}\right)
$$




$$
x_{2}=C_{2}^{I I I}+\frac{\left|\xi_{2}\right|}{2|\omega|}|Z| \sin \left(|\omega| t-\phi_{2}\right) .
$$

The kinetic energy term is negative-definite and consequently, $H^{I I I}$ is bounded from above. It seems that such a region has never been encountered and explored previously within the context of Hamiltonian system with balanced loss and gain. The system governed by the Hamiltonian $-H^{I I I}=H^{I}$ may be interpreted as that of a particle in the background of a Euclidean metric that is subjected to an effective external magnetic field $|\omega|$. The second order decoupled equations of motion resulting from $H^{I}$ and $H^{I I I}$ are identical, since they differ by an overall multiplication factor of -1 . However, the first order equations in these two regions are not identical. This is manifested in the fact that the time-dependent part of $x_{2}$ in Eqs. (38) and (40) differ by a sign. The solutions of the decoupled second order equations are identical in Region-I and RegionIII, if solved with identical initial conditions. It may be noted that under the transformation $B \rightarrow-B, \mathcal{D} \rightarrow \mathcal{D}^{T}$. Thus, the characteristic polynomial determining the solutions of Eq. (10) for $V=0$ is invariant under the transformation $B \rightarrow-B$. Consequently, even though $\mathcal{M}$ is not positive-definite for $B<0$, the solutions are periodic. A duality relation exists between the two Hamiltonians in Region-I and Region-III. In particular, the equations of motion of $H^{I}$ with potential $\mathcal{V}$ and that of $H^{I I I}$ with potential $-\mathcal{V}$ are the same. The duality relation may be used to find solutions in Region-III from that of Region-I and the vice verse.

A comment is in order before the end of this section. The solutions for $C \neq 0, \gamma \rightarrow 0$ can not be obtained from Eq. (38/39/40), since the limit is singular. However, the solutions of Eq. (10) with $\mathcal{D}$ given by Eq. (31) and $V=0$ may be obtained directly with a smooth limit $\gamma \rightarrow 0$ to the solutions of system with $C \neq 0$. In particular,

$$
x_{1}=|A| \cos \left(\omega t+\phi_{1}\right), \quad x_{2}=\sqrt{\frac{B-C}{B+C}}|A| \sin \left(\omega t+\phi_{2}\right),
$$

where the integration constant $A=|A| e^{i \phi_{1}}$ and the phase $\phi_{2}$ has the expression:

$$
\phi_{2}=\tan ^{-1}\left(\frac{\omega \sin \phi_{1}-2 \gamma \cos \phi_{1}}{\omega \cos \phi_{1}+2 \gamma \sin \phi_{1}}\right) .
$$

The center of the cyclotron motion is chosen to be at the origin by taking the remaining two integration constants equal to zero. The motion of the particle is confined along along $x_{1}$ for $B=C$ and $x_{2}$ diverges is for $B=-C$. Both of these cases $B= \pm C$ belong to Region-II, in which the solutions are diverging. The phases $\phi_{1}$ and $\phi_{2}$ become identical in the limit of vanishing gain and loss terms, i.e. $\gamma=0$. However, the amplitudes for the periodic solutions of $x_{1}$ and $x_{2}$ are different, leading to elliptical orbits. The solutions of the standard Landau Hamiltonian with circular orbits is recovered with a further choice of $C=0$. It may be noted that the limit $C \rightarrow 0, \gamma \rightarrow 0$ is also well-defined and independent of the order in which the limit has been taken.

\subsection{Quantum system}

The canonical quantization scheme is followed with $\hbar=1$ and the classical variables are treated as operators satisfying the relations:

$$
\left[x_{i}, x_{j}\right]=0,\left[p_{i}, p_{j}\right]=0,\left[x_{i}, p_{j}\right]=i \delta_{i j} .
$$

The canonical co-ordinate transformations (27) and Eq. (43) lead to the Heisenberg algebra in the new co-ordinate system:

$$
\left[\mathcal{X}_{i}, \mathcal{X}_{j}\right]=0,\left[\mathcal{P}_{i}, \mathcal{P}_{j}\right]=0,\left[\mathcal{X}_{i}, \mathcal{P}_{j}\right]=i \delta_{i j}
$$


The generalized momenta $\hat{\Pi}$ and commutation relation between its two components read,

$$
\hat{\Pi}_{1}=\mathcal{P}_{1}+\frac{|\omega|}{4} \mathcal{X}_{2}, \hat{\Pi}_{2}=\mathcal{P}_{2}-\frac{|\omega|}{4} \mathcal{X}_{1},\left[\hat{\Pi}_{1}, \hat{\Pi}_{2}\right]=i \frac{|\omega|}{2} .
$$

It is known [9, 10] for $B=0=C$ that balanced loss-gain systems can be formulated in terms of either Landau or symmetric gauge for the gauge potential giving rise to analogous magnetic field. The same can be generalized2 for $B \neq 0$ by taking the gauge potentials leading to real and analogous fields in the same gauge. Both the gauge potentials in Eq. (45) are taken in the symmetric gauge.

It was shown that the system defined by Eq. (5) has an effective description in terms of $H^{a}$ in Eq. (30). These two Hamiltonians are related by a canonical co-ordinate transformation defined in Eq. (27), which consists of a rotation from $X$ to $\hat{X}$, followed by a non-uniform scale transformation, $\hat{X} \rightarrow \mathcal{X}$. Thus, the eigenstates $\phi\left(x_{1}, x_{2}\right)$ in the $X$ co-ordinates are related to the eigenstates $\psi\left(\mathcal{X}_{1}, \mathcal{X}_{2}\right)$ in the $\mathcal{X}$ co-ordinates via a unitary transformation generated by the canonical co-ordinate transformations (27). In particular,

$$
\begin{aligned}
& \phi\left(x_{1}, x_{2}\right)=e^{i \hat{\mathcal{S}}} e^{i \theta \mathcal{J}_{3}} \psi\left(\mathcal{X}_{1}, \mathcal{X}_{2}\right), \\
& \hat{\mathcal{S}}:=\sqrt{\lambda_{+}}\left\{\mathcal{X}_{1}, \mathcal{P}_{1}\right\}+\sqrt{\lambda_{-}}\left\{\mathcal{X}_{2}, \mathcal{P}_{2}\right\}, \mathcal{J}_{3}:=\mathcal{X}_{1} \mathcal{P}_{2}-\mathcal{X}_{2} \mathcal{P}_{1}
\end{aligned}
$$

The operator $\mathcal{J}_{3}$ is the generator of rotation around an axis perpendicular to the ' $\mathcal{X}_{1}-\mathcal{X}_{2}$ 'plane. The operator $\hat{\mathcal{S}}$ generates scaling for $\mathcal{X}_{1}$ by an amount $\lambda_{+}{ }^{-\frac{1}{2}}$, for $\mathcal{X}_{2}$ by an amount $\lambda_{-}{ }^{-\frac{1}{2}}$. Similarly, it generates scaling for $\mathcal{P}_{1}$ by an amount $\sqrt{\lambda_{+}}$and for $\mathcal{P}_{2}$ by an amount $\sqrt{\lambda_{-}}$. The expectation value of an operator may be calculated either in terms of $\psi$ and $\phi$, since they are related through a unitary transformation. It should be noted that the unitary transformation is allowed even for non-vanishing potential, i.e. $V \neq 0$ and without any pre-specified symmetry on it. In particular, neither $\mathcal{J}_{3}$ nor $\hat{\mathcal{S}}$ is a symmetry of the system. Thus, an exactly solvable model in any of the two co-ordinate systems is also exactly solvable in the other co-ordinate system with seemingly different potentials.

\subsubsection{Region-I}

The matrix $\eta^{a}=I_{2}$ and $H$ reduces to the Landau Hamiltonian in the symmetric gauge. The eigen-value problem of the Landau Hamiltonian is well known[13. In particular, an operator $a$ and its adjoint $a^{\dagger}$ are defined as,

$$
a:=\frac{1}{\sqrt{|\omega|}}\left(\hat{\Pi}_{1}+i \hat{\Pi}_{2},\right), a^{\dagger}:=\frac{1}{\sqrt{|\omega|}}\left(\hat{\Pi}_{1}-i \hat{\Pi}_{2},\right),\left[a, a^{\dagger}\right]=1
$$

where the commutation relation between them allows to identify these two operators as annihilation and creation operators, respectively. The Hamiltonian and the eigenvalues can be expressed as,

$$
H^{(I)}=|\omega|\left(a^{\dagger} a+\frac{1}{2}\right), \quad E_{n}^{(I)}=\left(n+\frac{1}{2}\right)|\omega|, n \in \mathbb{Z}^{*} .
$$

The degenerate ground-state wave-functions for $C=0$ in terms of the co-ordinates $\left(x_{1}, x_{2}\right)$ have the form:

$$
\phi\left(x_{1}, x_{2}\right)=\left(\xi x_{1}-\xi^{*} x_{2}\right)^{m} e^{-\frac{|\omega|}{8}\left|\xi x_{1}-\xi^{*} x_{2}\right|^{2}}, \xi=\sqrt{\frac{2}{|\omega|}}\left(\sqrt{\left|\lambda_{+}\right|}+i \sqrt{\left|\lambda_{-}\right|}\right), m \in \mathbb{Z}^{*},
$$

\footnotetext{
${ }^{2}$ It is immaterial whether $C=0$ or not.
} 
where $\xi^{*}$ is the complex conjugate of $\xi$. Different values of $m$ correspond to linearly independent wave-functions $\phi\left(x_{1}, x_{2}\right)$ spanning the degenerate sub-space, since $E_{n}^{(I)}$ is independent of $m$. The most probable distribution of $\left|\phi\left(x_{1}, x_{2}\right)\right|^{2}$ is centered around an ellipse instead of a circle. The effect of the balanced loss and gain is to distort the circle around which the most probable distribution of the probability density occurs for the ground-state of the Landau Hamiltonian. This is consistent with the classical result. The annihilation and creation operators, when expressed in terms of $\left(x_{1}, x_{2}, p_{1}, p_{2}\right)$ have the following forms:

$$
a=\frac{1}{2}\left[\xi^{*}\left(p_{1}+\frac{1}{2} x_{2}\right)+\xi\left(p_{2}-\frac{1}{2} x_{1}\right)\right], \quad a^{\dagger}=\frac{1}{2}\left[\xi\left(p_{1}+\frac{1}{2} x_{2}\right)+\xi^{*}\left(p_{2}-\frac{1}{2} x_{1}\right)\right] .
$$

The excited states may be obtained by successive operations of the operator $a^{\dagger}$ on $\phi\left(x_{1}, x_{2}\right)$. One might define at this point a second set of creation and annihilation operators,

$$
b=\frac{1}{2}\left[\xi\left(p_{1}-\frac{1}{2} x_{2}\right)+\xi^{*}\left(p_{2}+\frac{1}{2} x_{1}\right)\right], \quad b^{\dagger}=\frac{1}{2}\left[\xi^{*}\left(p_{1}-\frac{1}{2} x_{2}\right)+\xi\left(p_{2}+\frac{1}{2} x_{1}\right)\right]
$$

which satisfy the commutation relation $\left[b, b^{\dagger}\right]=1$. It may be noted that $[a, b]=\left[a, b^{\dagger}\right]=0$ and similarly, $\left[a^{\dagger}, b^{\dagger}\right]=\left[a^{\dagger}, b\right]=0$ implying that $\left[H^{I}, b\right]=\left[H^{I}, b^{\dagger}\right]=0$. The operator $\mathcal{J}_{3}$, when expressed in the original co-ordinate, has the following expression:

$$
\begin{aligned}
\mathcal{J}_{3} & =b^{\dagger} b-a^{\dagger} a \\
& =\frac{1}{2}|\xi|^{2} J_{3}+\frac{1}{4}\left(\xi^{2}+\xi^{* 2}\right)\left(x_{1} p_{1}-x_{2} p_{2}\right)
\end{aligned}
$$

where $J_{3}:=x_{1} p_{2}-x_{2} p_{1}$ is the angular momentum operator in the original co-ordinate. The Hamiltonian $H$ is not invariant under a rotation on the $x_{1}-x_{2}$-plane due to the simultaneous presence of gauge potentials corresponding to the 'anomalous magnetic field' as well as external magnetic field. Thus, $\mathcal{J}_{3}$ and $J_{3}$ are not identical. The operator $\mathcal{J}_{3}$ satisfies the following commutation relations:

$$
\left[H^{I}, \mathcal{J}_{3}\right]=0, \quad\left[\mathcal{J}_{3}, b\right]=-b,\left[\mathcal{J}_{3}, b^{\dagger}\right]=b^{\dagger},\left[\mathcal{J}_{3}, a\right]=a,\left[\mathcal{J}_{3}, a^{\dagger}\right]=-a^{\dagger}
$$

The operator $b^{\dagger}$ acting on the wave-function (49) increase the angular-momentum eigen-value by one unit without changing the energy eigenvalue. On the other hand, the operator $a^{\dagger}$ s increases the energy eigen-value by one unit, while decreases the angular momentum eigen-value by one unit. Similarly, $b$ decreases the angular momentum eigen-value by one unit without changing the energy eigen-value, while $a$ decreases the energy eigen-value by one unit and increases the angular momentum eigen value by one unit. The number of degenerate states within a finite geometry is proportional to the magnetic flux piercing through the area. Thus, the number of degenerate states for a fixed geometry gets reduced in presence of the loss-gain terms.

\subsubsection{Region-II}

The Hamiltonian is not positive-definite. The analysis of the system in this region is well suited in the Landau gauge. The Hamiltonian in the Landau gauge may be obtained via appropriate unitary transformation [9]. In particular, a translational invariant Hamiltonian $H_{L_{1}}$ along the $x_{2}$ direction may be obtained as,

$$
H_{L_{1}}=\mathcal{G}^{-1} H^{I I} \mathcal{G}=\mathcal{P}_{1}^{2}-\left(\mathcal{P}_{2}-\frac{|\omega|}{2} \mathcal{X}_{1}\right)^{2}, \quad \mathcal{G}=e^{-i \frac{|\omega|}{4} \mathcal{X}_{1} \mathcal{X}_{2}}
$$


Similarly, a translational invariant Hamiltonian along the $\mathcal{X}_{1}$ direction may also be obtained. The method of separation of variables may be used to cast the eigen-value problem solely in terms of $\mathcal{X}_{1}, \mathcal{P}_{1}$. In particular,

$$
\psi_{0}^{-1} H_{L_{1}} \psi_{0}=\mathcal{P}_{1}^{2}-\frac{|\omega|^{2}}{4}\left(\mathcal{X}_{1}-\frac{2 k_{2}}{|\omega|}\right)^{2}, \quad \psi_{0}=e^{i k_{2} \mathcal{X}_{2}}
$$

The original eigenvalue-problem reduces to that of a particle moving in an inverted oscillator potential with shifted origin. There are no bound states.

\subsubsection{Region-III}

The Hamiltonian $H^{I I I}=-H^{I}$ is negative definite and bounded from above. Thus, the eigenvalue problem for $-H^{I I I}$ and $H^{I}$ are identical.

\subsection{Hall Effect}

The discussions so far have been confined to Landau Hamiltonian with a vanishing potential $V$. A description of the Hall effect requires an external uniform electric field. The potential $V$ is chosen as,

$$
V\left(x_{1}, x_{2}\right)=-\frac{E}{\omega^{2}}\left(B x_{1}-\gamma x_{2}\right), \omega^{2}=\left(B^{2}-\gamma^{2}\right) .
$$

The coupling of the velocity dependent non-Lorentzian interaction $C$ is taken to be zero for simplicity. The matrix $\mathcal{M}$ is singular for $B= \pm \gamma$ and the Hamiltonian formulation is based on the assumption of non-singular $\mathcal{M}$. Thus, the condition $B \neq \pm \gamma$ should be imposed in order to make it consistent with the Hamiltonian formulation. The Eq. (10) for this choice of $V$ and $C$ has the expression,

$$
\ddot{x}_{1}+\gamma \dot{x_{1}}-B \dot{x}_{2}=E, \quad \ddot{x}_{2}-\gamma \dot{x_{2}}+B \dot{x}_{1}=0,
$$

where the representation of $\mathcal{M}$ and $\mathcal{D}$ are given by Eq. (31). Eq. (57) describes Hall effect with balanced loss and gain, where $E$ is the magnitude of the external electric field along positive $x_{1}$-direction. A transformation $E \rightarrow-E$ allows to flip the direction of the electric field to the negative $x_{1}$-direction. The analysis of the system is presented in Region-I only.

The equations of motion in the transformed co-ordinate system have the form,

$$
\begin{aligned}
& \ddot{\mathcal{X}}_{1}-|\omega| \dot{\mathcal{X}}_{2}=\mathcal{E}_{1}, \ddot{\mathcal{X}}_{2}+|\omega| \dot{\mathcal{X}}_{1}=\mathcal{E}_{2}, \\
& \mathcal{E}_{1} \equiv \frac{E}{2 \sqrt{2 \lambda_{+}}}, \mathcal{E}_{2} \equiv-\frac{E}{2 \sqrt{2 \lambda_{-}}},
\end{aligned}
$$

implying that the effective electric field $\overrightarrow{\mathcal{E}}=\mathcal{E}_{1} \hat{\mathcal{X}}_{1}+\mathcal{E}_{2} \hat{\mathcal{X}}_{2}$ has non-vanishing components along both the directions. The Hall current is in the transverse direction to the external electric field $\overrightarrow{\mathcal{E}}$. However, the inverse transformation from $\left(\mathcal{X}_{1}, \mathcal{X}_{2}\right)$ to $\left(x_{1}, x_{2}\right)$ involves unequal scaling of the co-ordinates followed by a rotation, resulting in a Hall current along a direction making an angle $\phi_{H}=\tan ^{-1}\left(\frac{B}{\gamma}\right)$ with the direction of the external electric field $\vec{E}=|E| \hat{x}_{1}$. In particular, the solutions of Eq. (57) are,

$$
\begin{aligned}
& x_{1}=C_{1}^{I}+\frac{\left|\xi_{1}\right|}{2|\omega|}|Z| \cos \left(|\omega| t-\phi_{1}\right)-\frac{E \gamma}{\omega^{2}} t \\
& x_{2}=C_{2}^{I}-\frac{\left|\xi_{1}\right|}{2|\omega|}|Z| \sin \left(|\omega| t-\phi_{2}\right)-\frac{E B}{\omega^{2}} t
\end{aligned}
$$


It is interesting to note that the direction of the Hall current is not perpendicular to the direction of the applied electric field in presence of balanced loss and gain. Further, the angle between the direction of the Hall current and that of the external electric field depends on the ratio of the applied external magnetic field and the loss-gain parameter. It is known that similar behaviour for the Hall current is observed in plasma when the Hall parameter is very high.

The quantum Hamiltonian in presence of the external electric field is not rotationally invariant:

$$
H=\hat{\Pi}^{T} \hat{\Pi}+\mathcal{V}\left(\mathcal{X}_{1}, \mathcal{X}_{2}\right), \mathcal{V}\left(\mathcal{X}_{1}, \mathcal{X}_{2}\right)=-\frac{1}{2}\left(\mathcal{E}_{1} \mathcal{X}_{1}+\mathcal{E}_{2} \mathcal{X}_{2}\right)
$$

A rotation on the $\left(\mathcal{X}_{1}-\mathcal{X}_{2}\right)$-plane by an angle $\theta_{1}=-\tan ^{-1}\left(\frac{\mathcal{E}_{2}}{\mathcal{E}_{1}}\right)$, followed by unitary transformations cast the Hamiltonian $H$ as that of a one dimensional harmonic oscillator with shifted origin:

$$
\begin{aligned}
H_{1} & =\psi_{0}^{-1} \mathcal{G}^{-1} e^{i \theta_{1} \mathcal{J}_{3}} H e^{-i \theta_{1} \mathcal{J}_{3}} \mathcal{G} \psi_{0} \\
& =\mathcal{P}_{1}^{2}+\frac{|\omega|^{2}}{4}\left(\mathcal{X}_{1}-\frac{2 k_{2}}{|\omega|}-\frac{|\mathcal{E}|}{|\omega|^{2}}\right)^{2}-\frac{1}{4|\omega|^{2}}\left(\mathcal{E}^{2}+4 k_{2}|\omega||\mathcal{E}|\right)
\end{aligned}
$$

The rotation keeps the $\hat{\Pi}^{T} \hat{\Pi}$ term invariant, while mixing the components of $\overrightarrow{\mathcal{E}}$ such that it has non-vanishing components only along $\mathcal{X}_{1}$ in the rotated co-ordinate. The operator $\mathcal{G}$ transforms $H$ in rotational invariant gauge to $H$ in Landau gauge with translational invariance along $\mathcal{X}_{2}$ direction. The wave-function $\psi_{0}$ is used to decouple the kinetic energy part involving $\mathcal{P}_{2}$. The energy eigenvalues are

$$
E_{n, k_{2}}=\left(n+\frac{1}{2}\right)|\omega|-\frac{1}{4|\omega|^{2}}\left(\mathcal{E}^{2}+4 k_{2}|\omega||\mathcal{E}|\right),
$$

while the eigenfunctions are that of one dimensional harmonic oscillator in terms of the shifted co-ordinate $\tilde{\mathcal{X}}_{1}=\mathcal{X}_{1}--\frac{2 k_{2}}{|\omega|}-\frac{|\mathcal{E}|}{|\omega|^{2}}$. The probability current $\vec{J}$ has non-vanishing components only along $\mathcal{X}_{2}$ direction, i.e. the transverse direction to the direction of the applied electric field. In particular,

$$
J_{1}=0, \quad J_{2}=2 \rho\left(k_{2}-\frac{|\omega|}{2} \mathcal{X}_{1}\right),
$$

where $\rho$ is the probability density. However, when expressed in terms of the original co-ordinates $\left(x_{1}, x_{2}\right)$, the probability current has non-vanishing components along the directions of both $x_{1}$ and $x_{2}$, i. e. the direction of the external electric field and its transverse direction, respectively. This is consistent with the classical result.

\subsection{Spin, Pauli equation \& Supersymmetry}

Landau Hamiltonian with spin degrees of freedom for the particle contains an additional term due to the interaction between its magnetic moment and the external magnetic field. This gives rise to Pauli equation and appears in diverse branches of physics. A remarkable property of the Pauli equation is that it has an underlying supersymmetry[14. In this section, Landau Hamiltonian with balanced loss/gain term is generalized by including the spin degrees of freedom for the particle and shown to admit underlying supersymmetry. The supersymmetric Hamiltonian $H_{S}$ in Region- $\mathrm{I}(|B|>\gamma)$ is taken as,

$$
H_{S}=\frac{B}{2}\left(\Pi_{1}^{2}+\Pi_{2}^{2}\right)+\frac{\gamma}{2}\left\{\Pi_{1}, \Pi_{2}\right\}+\frac{|\omega|}{2} \sigma_{z},
$$


where the co-efficient of the spin-dependent term is chosen such that $H_{S}$ is supersymmetric. It may be noted that the Zeeman energy contains the effective magnetic field $|\omega|=B \sqrt{1-\left(\frac{\gamma}{B}\right)^{2}}$ instead of the external magnetic field $B$. This is essential in order to have underlying supersymmetry in $H_{S}$. This may also be interpreted as that the spin interacts with the external magnetic field $B$, but, the Landé $g$-factor is modified by a multiplicative factor of $\sqrt{1-\left(\frac{\gamma}{B}\right)^{2}}$ which reduces to its standard value in the limit $\gamma \rightarrow 0$. It is known that $H^{I I I}$ is negative-definite and does not admit supersymmetric generalizations. Similarly, $H^{I I}$ is not semi-positive definite and excludes the possibility of supersymmetric generalizations.

The supersymmetric Hamiltonian $H_{S}$ can be expressed in terms of the canonically transformed momenta $\left(\hat{\Pi}_{1}, \hat{\Pi}_{2}\right)$ as,

$$
H_{S}=\hat{\Pi}_{1}^{2}+\hat{\Pi}_{2}^{2}+\frac{|\omega|}{2} \sigma_{z},
$$

which has the standard form of the Pauli Hamiltonian. It is known that the Pauli Hamiltonian in Eq. 65 has a supersymmetric factorization, which can be used to introduce supercharges for $H_{S}$ in terms of $\Pi_{1}$ and $\Pi_{2}$. In particular,

$$
\begin{aligned}
& q_{1}=\sigma_{x} \hat{\Pi}_{1}-\sigma_{y} \hat{\Pi}_{2}=\sqrt{\frac{\lambda_{+}}{2}}\left(\Pi_{1}+\Pi_{2}\right) \sigma_{x}-\sqrt{\frac{\lambda_{-}}{2}}\left(\Pi_{1}-\Pi_{2}\right) \sigma_{y}, \\
& q_{2}=\sigma_{x} \hat{\Pi}_{2}+\sigma_{y} \hat{\Pi}_{1}=-\sqrt{\frac{\lambda_{-}}{2}}\left(\Pi_{1}-\Pi_{2}\right) \sigma_{x}+\sqrt{\frac{\lambda_{+}}{2}}\left(\Pi_{1}+\Pi_{2}\right) \sigma_{y}
\end{aligned}
$$

which satisfy the relations $\left\{q_{a}, q_{b}\right\}=2 \delta_{a b} H_{S}, a, b=1,2$. It should be mentioned here that another set of two supercharges satisfying the relations $\left\{Q_{a}, Q_{b}\right\}=2 \delta_{a b} H_{S}$ may be defined as,

$$
\begin{aligned}
Q_{1} & =\left(\sigma_{x} \Pi_{1}+\sigma_{y} \Pi_{2}\right) \sqrt{\frac{B}{2}} \cos \tilde{\theta}+\left(\sigma_{x} \Pi_{2}+\sigma_{y} \Pi_{x}\right) \sqrt{\frac{B}{2}} \sin \tilde{\theta} \\
& =\frac{\hat{\Pi}_{1}}{2} \sqrt{\frac{B}{\lambda_{+}}}\left(\sigma_{x}+\sigma_{y}\right)(\cos \tilde{\theta}+\sin \tilde{\theta})-\frac{\hat{\Pi}_{2}}{2} \sqrt{\frac{B}{\lambda_{-}}}\left(\sigma_{x}-\sigma_{y}\right)(\cos \tilde{\theta}-\sin \tilde{\theta}) \\
Q_{2} & =\left(\sigma_{x} \Pi_{1}-\sigma_{y} \Pi_{2}\right) \sqrt{\frac{B}{2}} \sin \tilde{\theta}+\left(\sigma_{x} \Pi_{2}-\sigma_{y} \Pi_{x}\right) \sqrt{\frac{B}{2}} \cos \tilde{\theta} \\
& =\frac{\hat{\Pi}_{1}}{2} \sqrt{\frac{B}{\lambda_{+}}}\left(\sigma_{x}-\sigma_{y}\right)(\cos \tilde{\theta}+\sin \tilde{\theta})+\frac{\hat{\Pi}_{2}}{2} \sqrt{\frac{B}{\lambda_{-}}}\left(\sigma_{x}+\sigma_{y}\right)(\cos \tilde{\theta}-\sin \tilde{\theta})
\end{aligned}
$$

where $\tilde{\theta}=-\frac{1}{2} \tan ^{-1} \frac{\gamma}{|\omega|}$. It should be noted here that all the four supercharges $\left(Q_{1}, Q_{2}, q_{1}, q_{2}\right)$ taken together do not give rise to $\mathcal{N}=4$ supersymmetry. Each set of supercharges $\left(Q_{1}, Q_{2}\right)$ and $\left(q_{1}, q_{2}\right)$ corresponds to $\mathcal{N}=2$ supersymmetry only. The factorization of the Hamiltonian is achieved in two different ways. The eigen-value problem and the state-space structure of $H_{S}$ in Eq. (65) is well known 14 and is not reproduced here.

\section{Summary \& Discussions}

It has been shown that the kinetic energy term for the Hamiltonian systems with balanced loss and gain may be made to be positive-definite by including a Lorentz interaction in the system. The result is quite general and applicable to a large class of systems with space-dependent lossgain terms. A few representations of the matrices appearing in the definition of the Hamiltonian is given with the identification of the regions in the parameter space of the theory in which the 
kinetic energy term is positive-definite. The presence of Lorentz interaction allows the balancing of loss-gain terms to occur in as many ways as the solutions of $\operatorname{Tr}(\mathcal{D})=0$ can be realized. The pair-wise balancing of loss-gain terms, which is a necessity in absence of the Lorentz interaction, appears as a special case in the present situation.

One important aspect of the present formulation is that the Hamiltonian system with balanced loss and gain may be interpreted as defined in the background of a metric without any loss-gain terms. The absence of loss-gain terms is manifested in modifying the magnitude of the external magnetic field due to the Lorentz force as well as coupling constants of various velocity-dependent non-Lorentzian interaction terms. The specific signature of the background metric depends on the form of the Hamiltonian defining the system. The effective Hamiltonian for the case of background Euclidean metric contains only Lorentz interaction with modified magnitude.

The classical and the quantum Landau Hamiltonian with balanced loss and gain have been studied in some detail. There are three regions in the parameter space depending on the nature of the background metric in the effective description of the system. The background metric is Euclidean provided the magnitude of the effective magnetic field is less than the magnitude of the applied magnetic field and the metric is pseudo-Euclidean, otherwise. The classical equations of motion are solved exactly in all three regions with periodic solutions in Region-I and RegionIII, which correspond to positive-definite and negative-definite background metric, respectively. In these two regions, the particle moves in an elliptic orbit with a cyclotron frequency that is less than its value in absence of loss-gain terms. There are no periodic solutions in RegionII corresponding to a background pseudo-Euclidean metric. The quantum bound states are obtained in Region-I and Region-III, consistent with the classical description. The results of the standard Landau Hamiltonian are valid even in presence of balanced loss and gain with a reduced value of the cyclotron frequency in these two regions. The Region-II does not admit any bound state.

The Hall effect with balanced loss and gain has been studied by including an external uniform electric field to the Landau Hamiltonian. One very interesting result for this case is that the Hall current is not necessarily in the perpendicular direction to the applied external electric field. The Hall current has non-vanishing components along the direction of the external electric field as well as to its transverse direction. This result is valid at the classical as well as quantum level. Similar results are known to exist in plasma in case the Hall parameter, the ratio between electron cyclotron frequency and the electron-heavy-particle collision frequency, is high. For the case of balanced loss-gain system, the Hall angle depends on the ratio of the external magnetic field and the gain-loss parameter. Any possible connection between these two systems is worth exploring in future.

The Pauli equation with balanced loss and gain has been studied from the viewpoint of underlying supersymmetry in the system. It has been shown that the system admits $\mathcal{N}=2$ supersymmetry, if the Zeeman energy contains the effective magnetic field $|\omega|$ instead of the external magnetic field $B$. This Zeeman energy term may also be interpreted as interaction between the external magnetic field $B$ and the spin degrees of freedom, but, with a modified Landé $g$-factor due to the presence of loss-gain terms. The state-space structure and the spectra are identical with the standard Pauli Hamiltonian. However, inclusion of Dresselhaus and Rashba spin-orbit interactions in the Hamiltonian is expected to give significant results and such investigations will be pursued in future. 


\section{Acknowledgments}

This work is partly supported by a grant (SERB Ref. No. MTR/2018/001036) from the Science \& Engineering Research Board(SERB), Department of Science \& Technology, Govt. of India under the MATRICS scheme.

\section{Appendix-A: Representation of $\mathcal{M}, R$ and $\mathcal{D}$}

Several representations of $\mathcal{M}, R$ and $\mathcal{D}$ for the case of pair-wise balancing of loss and gain terms have been discussed in the main text. The purpose of this Appendix is to present two different representations of $\mathcal{M}, R$ and $\mathcal{D}$, where the balancing of loss-gain terms are not necessarily in a pair-wise fashion.

\subsection{Representation-I}

The $N \times N$ symmetric matrix $\mathcal{M}$ is given by,

$$
\mathcal{M}=p I_{N}+q T, \quad[T]_{i j}=\delta_{i+1, j}+\delta_{i, j+1}, p, q \in \Re .
$$

The matrix $\mathcal{M}$ has the eigenvalues,

$$
\lambda_{k}=p+2 q \cos \left(\frac{k \pi}{N+1}\right), k=1,2, \ldots, N,
$$

and is positive-definite for $p>2|q|$. The matrix $\hat{O}$ that diagonalizes $\mathcal{M}$ has the expression,

$$
[\hat{O}]_{i j}=\sqrt{\frac{2}{N+1}} \sin \left(\frac{i j \pi}{N+1}\right) .
$$

For a generic choice of the matrix $J$ and the anti-symmetric matrix $A, R$ is an antisymmetric matrix, $R^{T}=-R$. The matrix $\mathcal{D}$ has the following expression:

$$
[\mathcal{D}]_{i j}=p[R]_{i j}+q\left([R]_{i+1, j}+[R]_{i-1, j}\right),
$$

where $[R]_{N+1, j}$ and $[R]_{0, j}$ are taken to be zero. It is apparent from the expressions of the diagonal elements $[\mathcal{D}]_{i, i}=q\left([R]_{i+1, i}+[R]_{i-1, i}\right)$ and $[R]_{i j}=-[R]_{j i}$ that the balancing of loss/gain terms does not necessarily occur in a pair-wise fashion. This particular representation with $V=\frac{1}{2} X^{T} X$ corresponds to a chain of linear oscillators with nearest-neighbour interaction and balanced lossgain that is subjected to velocity mediated coupling among different degrees of freedom.

\subsection{Representations-II}

The $N \times N$ symmetric matrix $\mathcal{M}$ with its elements $[\mathcal{M}]_{i j}$ given by,

$$
[\mathcal{M}]_{i j}=p \delta_{i j}+q\left(1-\delta_{i, j}\right)
$$

has eigenvalues $p-q$ with multiplicity $N-1$ and $p+(N-1) q$. The matrix is positive-definite provided,

$$
p>0, \quad-\frac{p}{N-1}<q<p .
$$


For a generic antisymmetric matrix $R$, the matrix $\mathcal{D}$ has the expression:

$$
\left[\mathcal{D}_{S}\right]_{i j}=\frac{q}{2} \sum_{k=1}^{N}\left([R]_{k j}+[R]_{k i}\right), \quad\left[\mathcal{D}_{A}\right]_{i j}=(p+q)[R]_{i j}+\frac{q}{2} \sum_{k=1}^{N}\left([R]_{k j}-[R]_{k i}\right) .
$$

The balancing of gain-loss terms does not necessarily occur in a pair-wise fashion for this particular representation.

\section{References}

[1] B. Peng, S. K. Ozdemir, F. Lei, F. Monifi, M. Gianfreda, G. L. Long, S. Fan, F. Nori, C. M. Bender, and L. Yang, Paritytime-symmetric whispering-gallery microcavities, Nature Physics, 10394 (2014).

[2] C. M. Bender, M. Gianfreda, S. K. Ozdemir, B. Peng, and L. Yang, Twofold transition in PT-symmetric coupled oscillators, Phys. Rev. A 88, 062111 (2013).

[3] C. M. Bender, M. Gianfreda and S. P. Klevansky, Systems of coupled PT-symmetric oscillators, Phys. Rev A90, 022114 (2014).

[4] I. V. Barashenkov and M. Gianfreda, An exactly solvable $\mathcal{P} \mathcal{T}$-symmetric dimer from a Hamiltonian system of nonlinear oscillators with gain and loss, J. Phys. A: Math. Theor. 47, 282001(2014).

[5] T. Shah, R. Chattopadhyay, K. Vaidya, S. Chakraborty, Conservative perturbation theory for nonconservative systems, Phys. Rev. E 92, 062927 (2015).

[6] D. Sinha, P. K. Ghosh, PT-symmetric rational Calogero model with balanced loss and gain, Eur. Phys. J. Plus, 132: 460 (2017), arXiv:1705:03426.

[7] A. Khare, A. Saxena, Integrable oscillator type and Schrdinger type dimers, J. Phys. A: Math. Theor. 50, 055202 (2017).

[8] P. K. Ghosh and Debdeep Sinha, Hamiltonian formulation of systems with balanced lossgain and exactly solvable models, Annals of Physics 388, 276 (2018), Arxive:1707.01122.

[9] D. Sinha, P. K. Ghosh, On the bound states and correlation functions of a class of Calogerotype quantum many-body problems with balanced loss and gain, arXiv: 1709.09648.

[10] D. Sinha and P. K. Ghosh, Integrable coupled Liénard-type systems with balanced loss and gain, arXiv:1804.02366

[11] C. M. Bender, Making sense of non-Hermitian Hamiltonians, Rept. Prog. Phys. 70, 947(2007); Carl M. Bender and Philip D. Mannheim, No-ghost theorem for the fourth-order derivative Pais-Uhlenbeck oscillator model, Phys. Rev. Lett. 100, 110402(2008).

[12] A. Mostafazadeh, Pseudo-hermitian representation of quantum mechanics, Int. J. Geom. Methods Mod. Phys. 7, 1191 (2010), arXiv:0810.5643, A. Mostafazadeh, Imaginary-scaling versus indefinite-metric quantization of the Pais-Uhlenbeck oscillator, Phys. Rev. D84, 105018 (2011), arXiv: 1107.1874.

[13] Quantum Mechanics, L. Landau and E. Lifshitz. Quantum Mechanics (Non-relativistic theory). 3rd ed. Oxford: Pergamon Press, 1977. 
[14] E. D'Hoker and L. Vinet, Supersymmetry of the Pauli Equation in the Presence of a Magnetic Monopole, Phys. Lett. B137, 72 (1984); M. de Crombrugghe and V. Rittenberg, Supersymmetric Quantum Mechanics, Ann. Phys. 151, 99 1983; F. ooper, A. Khare, R. Musto and A. Wipf, Supersymmetry and the Dirac Equation, Ann. Phys., 187, 1(1988).

[15] H. Bateman, Phys. Rev. 38, 815 (1931).

[16] E. Celeghini, M. Rasetti, and G. Vitiello, Ann. Phys. (N.Y) 215, 156 (1992).

[17] V. S. Afraimovich, N. N. Verichev, and M. I. Rabinovich, Stochastic synchronization of oscillation in dissipative system, Radiophysics and Quantum Electronics, 29, 795(1986).

[18] M.V. Ivanchenko, G.V. Osipov, V.D. Shalfeev and J. Kurths, Synchronization of two nonscalar-coupled limit-cycle oscillators, Physica D 189, 8(2004).

[19] A. P. Kuznetsov and J. P. Roman, Properties of synchronization in the systems of nonidentical coupled van der Pol and van der Pol-Duffing oscillators:Broadband synchronization. Physica D 238, 1499(2009).

[20] B. P. Pandey and Mark Wardle, Hall magnetohydrodynamics of partially ionized plasmas, Mon. Not. R. Astron. Soc. 385, 22692278 (2008).

[21] L. Rodman, Products of symmetric and skew-symmetric Matrices, Linear and Multilinear Algebra, 43, 19(1997). 